\title{
Patient-Reported Outcomes After Multiple-Revision ACL Reconstruction: Good but Not Great
}

\author{
Ian D. Engler, M.D., Matthew J. Salzler, M.D., Andrew J. Wall, M.D., \\ William R. Johnson, M.D., Amun Makani, M.D., Margaret V. Shields, M.D., and \\ Thomas J. Gill, M.D.
}

\begin{abstract}
Purpose: To evaluate the patient-reported and objective functional outcomes of patients undergoing multiple-revision anterior cruciate ligament (ACL) reconstruction surgery. The secondary purpose was to determine failure rates and factors associated with failure, with a focus on posterior tibial slope. Methods: All patients who underwent a repeat revision ACL reconstruction with a single surgeon over a 13-year period were identified. Chart data were obtained, including radiographic findings, operative details and findings, and postoperative examination findings. Failure was defined as subjective instability with evidence of graft incompetence on physical examination and MRI. Patients completed the International Knee Documentation Committee Subjective Knee Evaluation Form (IKDC-SKF) and Tegner Activity Level Scale. Patients who had outcomes scores completed a minimum of 2 years postoperatively were included. Results: Fourteen patients were available for follow-up; 12 underwent secondary revision procedures, and 2 underwent tertiary revisions. Three patients $(21 \%)$ had subsequent failure of the revision graft with mean time to failure of 27 months. Posterior tibial slope was significantly higher in the failures than in the nonfailures (13.3; 95\% CI $10.1-16.6$ versus 10.1 ; 95\% CI 6.7-11.4; $P=0.049$ ). Eleven patients completed outcomes measures at a mean of 42 months postoperatively (range 24-79 months). The mean Tegner activity score was 6.3 at follow-up, compared with 8.3 prior to the original ACL injury. The mean IKDC-SKF score was 70 at follow-up. Conclusion: Multiple revision ACL reconstruction surgery appears to have reasonable functional outcomes but is associated with a relatively high failure rate. Activity level following repeat revision surgery is diminished compared to the preinjury state, but most patients are able to return to recreational sports. Level of Evidence: Therapeutic Study, Level IV.
\end{abstract}

A nterior cruciate ligament (ACL) reconstruction is one of the most common orthopaedic procedures performed today. ${ }^{1,2}$ The procedure has a successful track record, with good or excellent results in 75\%-90\% of patients. ${ }^{3,4}$ However, failures of primary reconstruction occur, with reported failure rates of $8 \%-10 \% .{ }^{3,5,6}$ Failures

From the Department of Orthopaedics, Tufts Medical Center, Boston, Massachusetts, U.S.A. (I.D.E., M.J.S., M.V.S.); Department of Orthopaedic Surgery, Massachusetts General Hospital, Boston, Massachusetts, U.S.A. (A.J.W., W.R.J., A.M.); and Boston Sports Medicine, Dedham, Massachusetts, U.S.A. (T.J.G.).

The authors report no conflicts of interest in the authorship and publication of this article. Full ICMJE author disclosure forms are available for this article online, as supplementary material.

Received December 30, 2019; accepted June 11, 2020.

Address correspondence to Matthew J. Salzler, M.D., Tufts Medical Center, Department of Orthopaedics, 800 Washington Street, Boston, MA 02111, U.S.A.E-mail:mjsalzler@hotmail.com

(C) 2020 THE AUTHORS. Published by Elsevier Inc. on behalf of the Arthroscopy Association of North America. This is an open access article under the CC BY-NC-ND license (http://creativecommons.org/licenses/by-nc-nd/4.0/).

2666-061X/206

https://doi.org/10.1016/j.asmr.2020.06.013 can result from traumatic injury or from atraumatic laxity, which can be due to nonanatomic graft positioning, failure of fixation or biologic failure with lack of graft incorporation. ${ }^{7-11}$ Patients with recurrent instability may benefit from revision ACL reconstruction. ${ }^{12-14}$

Revision surgery has inherent challenges, including postsurgical adhesions, bone loss, difficulty in achieving an uncompromised and anatomic tunnel location, the presence of previously placed hardware, increased reliance on allografts, and the need to address underlying associated pathology that may have led to failure. ${ }^{15}$ Results of single-revision reconstructions have been well studied and, generally, have been inferior to primary reconstructions, with failures rates 3 to 4 times higher than after primary reconstruction. ${ }^{13,14,16-20}$ Many of these failures are managed with multiple revision surgery, also known as repeat revision or re-revision surgery.

Although multiple studies exist on the outcomes of first-time revision ACL surgery, there is a paucity of literature on the outcomes of patients undergoing multiple revision surgery. As more ACL reconstructions are 
performed, and more people remain physically active later in life, the number of failed ACL reconstructions and the need for revision surgery is going to rise. Even after revision surgery, many patients choose to return to their sports and incur risk of repeat injury to their ACLreconstructed knee. To date, little has been reported on this challenging group of patients who have undergone multiple revision ACL reconstructions, and more study is warranted. ${ }^{3,21}$ This information is vital for surgeons as they counsel their patients, particularly as the risks and costs associated with repeat revision surgery can be higher due to graft choice, increased operative time, need for staged procedures, and use of specific implants.

The purpose of this study was to evaluate the patientreported and objective functional outcomes of patients undergoing multiple revision ACL reconstruction surgery. The secondary purpose was to determine failure rates and factors associated with failure, with a focus on posterior tibial slope. Our hypothesis was that multiple ACL reconstruction would have higher failure rates than primary ACL reconstruction and would have worse outcomes, but most patients would be able regain a reasonable level of function and return to recreational sports.

\section{Methods}

\section{Inclusion and Exclusion Criteria}

The study design was a retrospective case series. Electronic medical records were reviewed to identify all patients who underwent repeat revision ACL reconstruction following a failed previous revision reconstruction between 1999 and 2012 with the senior author (T.J.G.), a sports medicine-trained orthopedic surgeon, at a single institution. Exclusion criteria included patients younger than 18 years of age, associated osteotomy, multiligament reconstruction, or fewer than 2 years of follow-up. The surgeon performed no tibial deflexion osteotomies over this time period. This study was approved by the institutional review board at our institution.

\section{Surgical Procedure and Rehabilitation}

Indications for repeat revision ACL reconstruction included evidence of graft failure on physical examination and MRI alongside clinically relevant symptoms of instability. All revision reconstructions were performed arthroscopically. A transtibial technique was used if the anatomic femoral ACL footprint could be reached. ${ }^{22,23}$ If not, an accessory anteromedial portal was used..$^{24}$ In each case, the residual failed graft was debrided. Removal of femoral and tibial hardware from the primary ACL reconstruction was performed if it impeded proper tunnel placement. If the previous graft tunnel coincided with the planned graft tunnel, single-staged grafting was used on the tibial and/or femoral tunnels to fill the bony defect with either a Milagro biocomposite screw (Depuy Mitek,
Raynham, MA) or allograft bone graft. The planned tunnels were then drilled through these grafts. Two-stage reconstruction was not undertaken for any patients by the surgeon over this time period. The optimal graft for the patient was chosen after shared decision making with the patient, taking into consideration prior grafts used, available autografts, and patient age and activity level. Fixation of the revision ACL graft was secured with Guardsmen interference screws (Conmed, Utica, NY) or Milagro screws.

Postoperatively, patients were allowed partial weight bearing in a brace for 6 weeks. They were instructed to use a continuous passive motion machine for at least 10 hours per day for the first 2 weeks. Physical therapy commenced approximately 3 to 5 days after the procedure, with goals of regaining full muscle strength, cardiovascular conditioning, and return to sports-specific training. In general, strengthening began at 6 weeks, jogging began at 3.5 months, and return to sport was permitted, pending clearance of sport-specific testing, at around 6 to 8 months. Patients were cleared for sports once they passed functional performance testing and were able to perform sports-specific activities.

\section{Data Collection}

Demographics obtained included the date of initial injury, age, gender, laterality, mechanism of injury, body mass index (BMI), and physical examination findings. Surgical data obtained included the date of repeat revision ACL reconstruction, graft type, graft fixation methods, associated procedures, use of bone grafting, and meniscus and cartilage status. Other variables assessed included previous reconstruction graft types and dates of previous reconstructions. Postoperative graft failure was defined as subjective instability with evidence of graft incompetence on physical examination and MRI.

\section{Radiographs}

Knee radiographs were analyzed for tunnel placement from previous reconstructions as well as posterior tibial slope. Tibial tunnel positioning was calculated by measuring the center of the tunnel from the anterior edge of the plateau as a percentage of the total anteroposterior width of the tibial plateau (Fig 1). ${ }^{25}$ Posterior tibial slope was calculated as the angle between the perpendicular of the proximal tibial diaphyseal axis and the line between the most superior points of the anterior and posterior margins of the tibial plateau (Fig 2). ${ }^{26}$

\section{Outcome Scores}

The International Knee Documentation Committee Subjective Knee Evaluation Form (IKDC-SKF) and Tegner Activity Level Scale were collected via mail or telephone. ${ }^{27,28}$ Outcomes scores were used to assess the activity level of the patient prior to the initial ACL injury, after the primary reconstruction, and at final follow-up. 


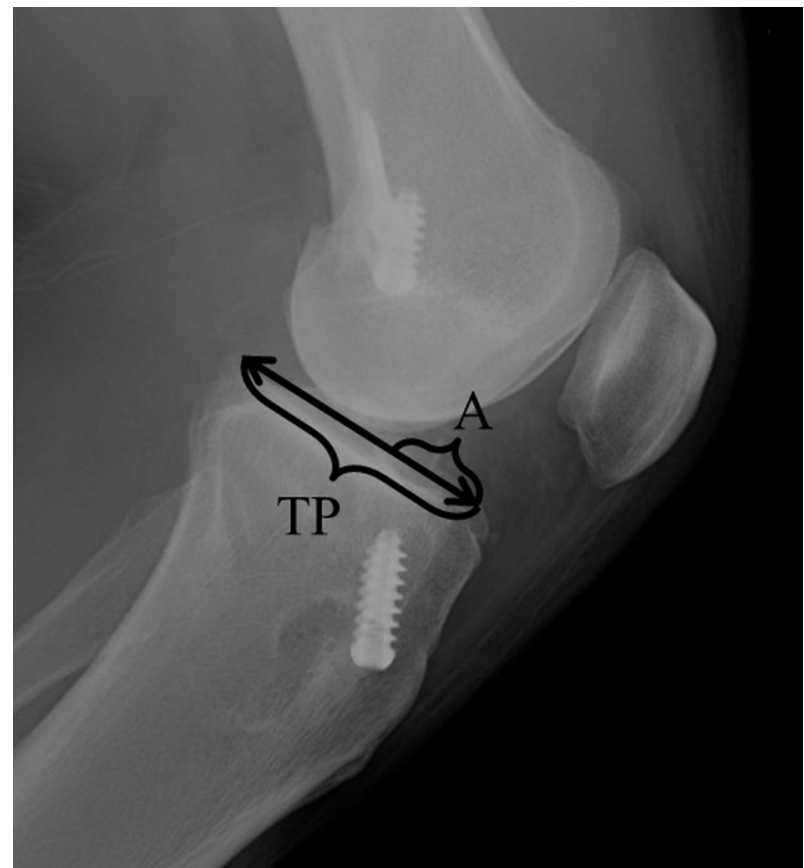

Fig 1. Tibial tunnel position. Tibial tunnel positioning was calculated by measuring the center of the tunnel from the anterior edge of the tibial plateau (A) as a percentage of the total anteroposterior tibial plateau distance (TP).

Mean IKDC-SKF scores were calculated for 3 subgroups of BMI-under 25, 25 to 30, and over 30-and by mechanism of injury-atraumatic versus traumatic.

\section{Statistics}

Continuous variables were described using means and ranges. Categorical variables were presented with absolute frequencies. A 2-sample $t$ test was used to compare preoperative and postoperative Tegner scores, IKDC-SKF score by mechanism of injury, preoperative and postoperative tibial tunnel anterior placement percentage, and tibial slope by subsequent failure versus no failure. Significance was set at $P<0.05$. Because of the small sample size, we also calculated $95 \%$ confidence intervals for posterior tibial slope to assess for reliability. Spearman correlations were used to evaluate the influence of the self-reported outcome on activity level.

\section{Results}

\section{Patient Population}

A total of 22 consecutive patients were initially identified. Fourteen patients were able to be contacted and consented to participate. Eleven patients completed these outcomes surveys at a minimum of 24 months postoperatively.

\section{Patient Demographics}

Demographics are displayed in Table 1. Eleven patients had follow-up outcome measures of at least 24 months (mean 42 months, range 24-79 months) since their repeat revision procedures. Of these, there were 10 females and 1 male. The average age at time of follow-up was 42.5 years (range 24-61 years). There were 8 left knee reconstructions and 3 right knee reconstructions. Seven patients had BMIs of less than 25, 3 had BMIs between 25 and 30, and 1 had a BMI greater than 30. Nine patients underwent second revision surgeries, and 2 patients underwent third revision surgeries. The prior grafts were patellar tendon allografts in 5 patients, Achilles allografts in 2 patients, a

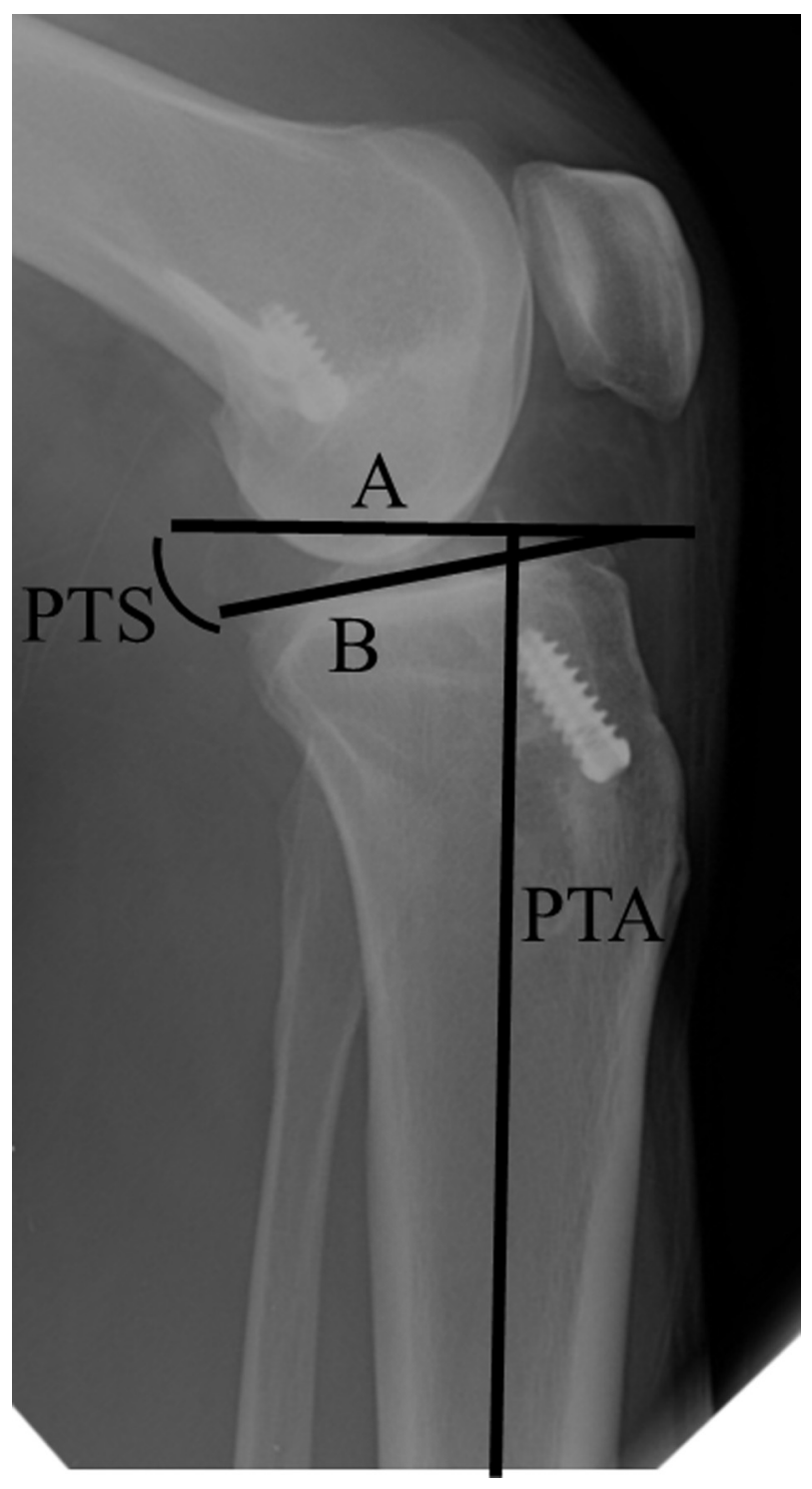

Fig 2. Posterior tibial slope. Posterior tibial slope was calculated as the angle (PTS) between the perpendicular (A) of the proximal tibial diaphyseal axis (PTA) and the line between the most superior points of the anterior and posterior margins of the tibial plateau (B). 
Table 1. Patient Demographics

\begin{tabular}{lc}
\hline Sex & $\mathrm{N}=$ \\
Male & 1 \\
Female & $\mathrm{N}=$ \\
Laterality & 3 \\
Right & 8 \\
Left & Years mean (range) \\
Age & $39.8(21-58)$ \\
At repeat revision reconstruction & $42.5(24-61)$ \\
At final follow up & $\mathrm{N}=$ \\
BMI & 7 \\
$<25 \mathrm{~kg} / \mathrm{m}^{2}$ & 4 \\
$>25 \mathrm{~kg} / \mathrm{m}^{2}$ & Months (range) \\
Time between procedures & $57(3-206)$ \\
Primary to $1^{\text {st }}$ revision & $78(21-151)$ \\
$1^{\text {st }}$ to $2^{\text {nd }}$ revision & 1 \\
Male & 10 \\
Female &
\end{tabular}

BMI, body mass index.

hamstring or patellar tendon autograft in 1 patient each, and unknown in 2 patients. The cause of graft failure prior to the repeat revision surgery was traumatic in 6 patients $(55 \%)$ and without clear traumatic event in 5 patients $(45 \%)$.

The average time from the primary procedure to first revision was 57 months (range 3-206 months). The average time from first revision to second revision was 78 months (range 21-151 months). Available graft type for all previous reconstructions was assessed (Table 2).

\section{Intraoperative Variables and Objective Outcomes}

In the repeat revision surgery, patellar tendon allograft was used in 7 patients, patellar tendon autograft was used in 3 patients, and hamstring tendon autograft was used in 1 patient (Table 2). A concomitant partial meniscectomy was performed in 11 patients, and a chondroplasty was performed in 5 patients. Eight patients required single-staged grafting of previous tunnels. Three patients required grafting of the femur only, 2 patients required grafting of the tibia, and 3 patients required grafting of both the tibia and femur. Femoral tunnel grafting was managed by occupying the previous tunnel with a biocomposite interference screw in 4 cases and a patella allograft plug in 2 cases. Tibial tunnel grafting was managed with a biocomposite screw in 4 cases and a patella allograft plug in 1 case.

There was no significant difference in preoperative and postoperative tibial tunnel placement following repeat revision ACL reconstruction. The mean preoperative tibial tunnel location was $30.3 \%$ of the anteroposterior tibial plateau distance (range 20.2\%$53.9 \%)$ compared with the postoperative location of $28.2 \%$ (range $12.4 \%-39.7 \%)(P=0.11)$.

The mean posterior tibial slope was 10.1 degrees. The failure group averaged $13.3^{\circ}$ (range $11^{\circ}-17 ; 95 \%$ CI 10.1-16.6), whereas the intact group averaged $9.1^{\circ}$ (range 3-13; 95\% CI 6.7-11.4). A $t$ test showed a significant difference in tibial slope between intact and failure groups $(P=0.049)$, though the confidence intervals overlapped.

All patients had range-of-motion data documented at least 1 year following surgery. Two patients had 125 degrees of flexion, 3 patients had 130 degrees of flexion, 5 patients had 135 degrees of flexion, and 1 patient had 140 degrees of flexion. All were able to extend the knee fully at time of last follow-up. All patients except the 3 failures described below had stable Lachman and pivot-shift examinations at the most recent postoperative visit.

\section{Complications}

No intraoperative or immediate postoperative complications occurred following the procedure. However,

Table 2. Graft Types by Surgical Procedure

\begin{tabular}{|c|c|c|c|c|}
\hline Case No. & Primary ACL Reconstruction & First Revision & Second Revision & $\begin{array}{l}\text { Third Revision } \\
\text { (if applicable) }\end{array}$ \\
\hline $\bar{l}$ & Unknown & Achilles Allo & PT Allo & \\
\hline 2 & Unknown & Unknown & PT Allo & \\
\hline 3 & Allograft (unspecified) & Achilles Allo & PT Allo & \\
\hline 4 & PT Auto & PT Auto & PT Allo & PT Allo \\
\hline 5 & Unknown & PT Allo & PT Allo & \\
\hline 6 & HT Auto & PT Allo & PT Auto & \\
\hline 7 & Unknown & PT Allo & PT Allo & \\
\hline 8 & Unknown & HT Auto & PT Allo & PT Auto \\
\hline 9 & PT Auto & Unknown & HT Auto & \\
\hline 10 & PT Allo & PT Allo & PT Allo & \\
\hline 11 & PT Allo & PT Allo & PT Auto & \\
\hline $12 *$ & HT Auto & PT Allo & PT Auto & PT Allo \\
\hline $13 *$ & HT Auto & Tibialis Anterior Allograft & PT Auto & PT Allo \\
\hline $14^{*}$ & Allograft (unspecified) & Allograft (unspecified) & PT Auto & PT Allo \\
\hline
\end{tabular}

Achilles Allo, Achilles tendon allograft; HT Auto, hamstring tendon autograft; PT Allo, patellar tendon allograft; PT Auto, patellar tendon autograft.

*Patients with graft failure during study period and subsequent revision. 
Table 3. Outcomes Scores

\begin{tabular}{|c|c|c|c|}
\hline Outcomes Score & Preinjury & $\begin{array}{c}\text { Post-primary } \\
\text { Reconstruction }\end{array}$ & Final Follow-up \\
\hline Tegner Scale & $8.3(3-10)$ & $7.1(5-9)$ & $6.3^{*}(5-9)$ \\
\hline IKDC-SKF & N/A & $\mathrm{N} / \mathrm{A}$ & $70(41-95)$ \\
\hline
\end{tabular}

NOTE. Data are presented as mean (range).

IKDC-SKF, International Knee Documentation Committee Subjective Knee Evaluation Form.

*Significantly different from preinjury score $(P=0.015)$.

during the study period, 3 of the 14 patients $(21.4 \%)$ had subsequent failure of the revision graft. All graft failures were attributable to a traumatic sports injury. These patients ranged in age from 20-23 years old at time of failure and included 2 males and 1 female. All had received patellar tendon autografts. The average time from re-revision reconstruction to failure was 27 months (range 17-41 months). All 3 patients elected to undergo another revision procedure, which was their third revision.

\section{Patient Reported Outcomes}

Patient-reported outcomes are presented in Table 3. Prior to any ACL injury, the mean Tegner activity score was 8.3 (range 3-10). After the first reconstruction, the average score was 7.1 (range 5-9). At the most recent follow-up, the mean Tegner score was 6.3 (range 4-8). There was a significant difference in Tegner activity score between preinjury and current states $(P=0.015)$. Only 2 patients $(18 \%)$ regained the same level of activity that they had had prior to their original injury. There was no significant difference in Tegner activity score between post-primary ACL reconstruction and current states $(P=0.22)$, though this was limited by the small sample size. Five patients $(45 \%)$ regained the same level of activity they had following their primary reconstructions.

The average IKDC-SKF score after the most recent revision reconstruction was 70 (range 41-95). For those who had BMIs under 25, the mean IKDC-SKF score was 73. For patients with BMIs of 25 to 30, the mean IKDCSKF score was 72. For the patient with a BMI greater than 30, the IKDC-SKF score was 41. Statistical comparison across BMI subgroups was prohibited by low power.

Mean IKDC-SKF scores following final revision were 76.6 in patients with traumatic injuries and 62.3 in those with atraumatic injuries $(P=0.10)$. Mean Tegner activity scores were 6.7 in the traumatic group and 5.8 in the atraumatic group $(P=0.31)$. Statistical comparison was limited by low power.

\section{Discussion}

In this study of multiple revision ACL reconstructions, we found reasonably good clinical outcomes at a mean of 42 months postoperatively, although there was a relatively high rate of recurrent graft failure. Of our 14 patients, $3(21.4 \%)$ failed within the study period. Griffith et al. also reported a high failure rate $(13 \%)$ following repeat revision reconstruction in 15 patients. ${ }^{3}$ They had a younger group, with a mean age of 27, compared to ours of 39.8, and a very similar proportion of traumatic mechanisms for prior revision graft failure. They used a similarly high rate of patellar tendon allograft, as in our study. Using a very different over-the-top technique for multiple revision reconstruction, Buda et al. reported a failure rate of $16.7 \%$ in 24 Italian male patients with a mean age of $31 .{ }^{29}$

Our 3 failures were all young (range 20-23 years old), and all received patellar tendon autografts in their multiple revision reconstruction because of their young age and a preference for autograft in younger patients. All failed due to traumatic mechanisms. Younger patients have been shown to have higher rates of graft failure after primary and revision ACL reconstruction, in part due to their higher rates of return to sport. ${ }^{30}$ It is unlikely that an accelerated rehabilitation program contributed to these failures because they failed 17-41 months after their reconstruction. ${ }^{31,32}$

Patient-reported outcomes, with a minimum followup of 2 years, showed a significant decline from preinjury state. We found a decrease in Tegner activity scale from a preinjury state of 8.3 to a current state of 6.3, which is greater than the minimally detectable change for the Tegner activity scale. ${ }^{33}$ This is comparable to other studies showing a decrease in prior activity level following repeat revision. ${ }^{34}$ A score of 6.3 correlates with recreational sports such as tennis and frequent jogging, showing that these patients can return to some level of sports. The average IKDC-SKF score of 70 was less than the patient-acceptable symptom state following primary ACL reconstruction of $75.9 .^{35}$ The patient-acceptable symptom state has not been defined for revision ACL reconstruction, much less for multiple ACL reconstruction, so we do not know whether this is an acceptable score for this population. All patients in our study were able to achieve functional knee motion of at least 125 degrees of flexion following the last revision surgery.

Anatomic susceptibility to ACL rupture in patients after multiple revision has gained interest because of the possibility of addressing these factors so as to decrease stress on the revision ACL graft. One such factor is posterior tibial slope. A meta-analysis by Wordeman et al. found that there was a significantly higher posterior tibial slope in patients with ACL injuries compared to those with uninjured ACLs, suggesting that the osseous anatomy of the tibia plays a role in rupture rates of the native ACL. ${ }^{36}$ Studies have shown significantly larger posterior tibial slope in patients who failed both primary reconstruction $\left(8.4^{\circ} \text { vs } 6.5^{\circ} \text { in nonfailures }\right)^{37}$ and revision ACL reconstruction $\left(13.2^{\circ}\right.$ vs 10.9). ${ }^{38}$ Some authors recommend considering an anterior closing wedge osteotomy if the posterior tibial slope exceeds $12^{\circ} .^{38}$ 
Less is known about the effect of posterior tibial slope on multiple-revision ACL surgery. Patients who have failed at least 2 reconstruction surgeries are particularly likely to have intrinsic factors leading to instability. Dejour et al. reviewed 9 patients undergoing second revision ACL reconstruction with an associated tibial deflexion osteotomy, decreasing the mean posterior tibial slope from $13.2^{\circ}$ preoperatively to $4.4^{\circ}$ postoperatively. ${ }^{39}$ With an average follow-up of 4 years, they found no complications or graft ruptures, suggesting that decreasing the tibial slope may lead to less stress on the ACL graft, thereby increasing the stability of the construct. Similarly, Sonnery-Cottet et al. followed, for an average of 31.6 months, 5 patients who underwent combined multiple revision ACL reconstruction with a proximal tibial anterior closing wedge osteotomy and found satisfactory outcomes after decreasing the posterior tibial slope from $13.6^{\circ}$ preoperatively to $9.2^{\circ}$ postoperatively. ${ }^{40}$

In our study, the average posterior tibial slope in patients who failed was $13.3^{\circ}$ versus $9.1^{\circ}$ for those who did not fail. This was a statistically significant difference by our a priori definition of $P<0.05$, showing the effect of increased posterior tibial slope on risk of ACL graft rupture. The fact that the $95 \%$ confidence intervals overlap shows that this finding does not have a high degree of reliability. There remains no definitive degree of posterior tibial slope that puts a patient at an increased risk of failure, ${ }^{36}$ but agreement is building for consideration of an osteotomy for patients with posterior tibial slopes equal to or greater than $12^{\circ} .^{39,41}$

All 11 patients with intact grafts at final follow-up required partial meniscectomies, and 5 required partial chondroplasty at the time of last revision surgery. High rates of chondral pathology and meniscal tears with multiple revision ACL reconstruction have also been shown by Wegrzyn et al., who noted 11 medial and lateral meniscal tears in their series of 10 patients undergoing second revision ACL reconstruction surgery. ${ }^{42}$ Meniscal pathology has been shown to increase the risk of poor outcomes ${ }^{43,44}$ and graft rupture in the setting of revision ACL reconstruction. ${ }^{30}$ Cartilage pathology has also been shown to worsen outcomes in this setting, with increased pain, stiffness and functional limitation. ${ }^{43,44}$

We did not identify a significant difference in outcome scores when comparing a traumatic versus atraumatic mechanism of prior graft failure, though mean IKDCSKF scores were 76.6 and 62.3, respectively. However, our population was underpowered to detect such a difference. Prior studies have shown improved outcomes after re-revision ACL reconstruction in patients with traumatic graft rupture. ${ }^{3,29}$

Prior research has been done on outcomes in multiple revision ACL reconstruction. Liechti et al. performed a systematic review of multiple revision ACL reconstruction to evaluate patient outcomes, risk factors and concomitant knee injuries. ${ }^{34}$ They identified 6 studies $(\mathrm{N}=214)$ meeting their criteria, including 1 case-control study (level III study) and 5 case series (level IV studies). They found that patient outcomes after re-revision ACL reconstruction improved compared to preoperative scores but remained inferior to those following primary or revision ACL reconstruction. The 3 included studies that statistically analyzed preoperative versus postoperative IKDC scores showed significant improvement (preoperative range of 39.5-59, postoperative range of 79.1-81.3). ${ }^{3,29,40}$ Although that study helped to identify general outcomes after multiple revision surgery, the studies within it were heterogeneous, and each included associated ligamentous or bony procedures. As the authors state, only 2 studies reported failure rates, 2 evaluated posterior tibial slope, and 2 included staged procedures for femoral or tibial tunnel widening. All studies included 1 or more patients with concurrent osteotomy or ligamentous reconstruction.

Our study differs from this systematic review because it follows the experience of a single surgeon, with a consistent surgical technique and rehabilitation protocol, without associated ligamentous or bony procedures. In addition, preoperative tibial slope was calculated, adding to the body of literature on the matter. Finally, all multiple revision reconstructions performed by this surgeon over the time period were single-stage reconstructions, nearly entirely with stacked biocomposite screws to account for bone loss. This shows the versatility of the stacked-screws construct in revision ACL reconstruction in the setting of bone loss.

There is increasing interest in additional procedures such as high tibial osteotomy (HTO) or anterolateral ligament (ALL) reconstruction to supplement stability of the knee in ACL reconstruction, particularly in revision settings. ${ }^{29,45}$ Neither HTO nor ALL reconstruction was performed in any of our revisions, yet the outcomes of our group were largely positive. This shows that multiple revision ACL reconstruction can be performed safely without these supplemental procedures. That said, the failures had a nonsignificantly increased posterior tibial slope, suggesting possible benefit of an HTO. Further study is warranted to compare directly HTO and ALL reconstruction alongside multiple-revision ACL reconstruction versus ACL reconstruction alone.

\section{Limitations}

The greatest limitation to this study is the small sample size. We were underpowered to identify a difference in outcome scores when comparing, for example, revision graft type, gender or need for bone grafting. This study is also subject to selection bias, as minimum 2-year patient-reported outcomes measures were able to be obtained in only $50 \%$ of patients. 


\section{Conclusions}

Multiple revision ACL reconstruction surgery appears to have reasonable functional outcomes but is associated with a relatively high failure rate. Activity level following repeat revision surgery is diminished compared to the preinjury state, but most patients are able to return to recreational sports.

\section{References}

1. Bollen S, Scott B. Rupture of the anterior cruciate ligament-a quiet epidemic? Injury 1996;27:407-409.

2. Lyman S, Koulouvaris P, Sherman S, et al. Epidemiology of anterior cruciate ligament reconstruction: Trends, readmissions, and subsequent knee surgery. J Bone Joint Surg Am 2009;91:2321-2328.

3. Griffith TB, Allen BJ, Levy BA, et al. Outcomes of repeat revision anterior cruciate ligament reconstruction. Am J Sports Med 2013;41:1296-1301.

4. Freedman KB, D'Amato MJ, Nedeff DD, et al. Arthroscopic anterior cruciate ligament reconstruction: A metaanalysis comparing patellar tendon and hamstring tendon autografts. Am J Sports Med 2003;31:2-11.

5. Carlisle JC, Parker RD, Matava MJ. Technical considerations in revision anterior cruciate ligament surgery. J Knee Surg 2007;20:312-322.

6. Grossman MG, ElAttrache NS, Shields CL, Glousman RE. Revision anterior cruciate ligament reconstruction: Threeto nine-year follow-up. Arthroscopy 2005;21:418-423.

7. Carson EW, Anisko EM, Restrepo C, et al. Revision anterior cruciate ligament reconstruction-etiology of failures and clinical results. J Knee Surg 2004;17:127-132.

8. Chen JL, Allen CR, Stephens TE, et al. Differences in mechanisms of failure, intraoperative findings, and surgical characteristics between single-and multiple-revision ACL reconstructions: A MARS cohort study. Am J Sports Med 2013;41:1571-1578.

9. Denti M, Vetere DL, Bait C, et al. Revision anterior cruciate ligament reconstruction: Causes of failure, surgical technique, and clinical results. Am J Sports Med 2008;36: 1896-1902.

10. Johnson D, Fu F. Anterior cruciate ligament reconstruction: Why do failures occur? Instr Course Lect 1995;44:391.

11. Group M. Effect of graft choice on the outcome of revision anterior cruciate ligament reconstruction in the Multicenter ACL Revision Study (MARS) cohort. Am J Sports Med 2014;42:2301-2310.

12. George MS, Dunn WR, Spindler KP. Current concepts review: Revision anterior cruciate ligament reconstruction. Am J Sports Med 2006;34:2026-2037.

13. Johnson DL, Swenson TM, Irrgang JJ, et al. Revision anterior cruciate ligament surgery: Experience from Pittsburgh. Clin Orthop Relat Res 1996;325:100-109.

14. Uribe JW, Hechtman KS, Zvijac JE, et al. Revision anterior cruciate ligament surgery: Experience from Miami. Clin Orthop Relat Res 1996;325:91-99.

15. Group M. Descriptive epidemiology of the Multicenter ACL Revision Study (MARS) cohort. Am J Sports Med 2010;38:1979-1986.
16. Wright RW, Gill CS, Chen L, et al. Outcome of revision anterior cruciate ligament reconstruction: A systematic review. J Bone Joint Surg Am 2012;94:531-536.

17. Noyes FR, Barber-Westin SD. Revision anterior cruciate ligament surgery: Experience from Cincinnati. Clin Orthop Relat Res 1996;325:116-129.

18. Wirth CJ, Kohn D. Revision anterior cruciate ligament surgery: Experience from Germany. Clin Orthop Relat Res 1996;325:110-115.

19. Kamath GV, Redfern JC, Greis PE, Burks RT. Revision anterior cruciate ligament reconstruction. Am J Sports Med 2011;39:199-217.

20. Wright RW, Dunn WR, Amendola A, et al. Anterior cruciate ligament revision reconstruction: Two-year results from the MOON cohort. J Knee Surg 2007;20: 308-311.

21. Wegrzyn J, Chouteau J, Philippot R, et al. Repeat revision of anterior cruciate ligament reconstruction: A retrospective review of management and outcome of 10 patients with an average 3-year follow-up. Am J Sports Med 2009;37:776-785.

22. Bhatia S, Korth K, Van Thiel GS, et al. Effect of reamer design on posteriorization of the tibial tunnel during endoscopic transtibial anterior cruciate ligament reconstruction. Am J Sports Med 2013;41:1282-1289.

23. Rue JP, Ghodadra N, Bach BR Jr. Femoral tunnel placement in single-bundle anterior cruciate ligament reconstruction: A cadaveric study relating transtibial lateralized femoral tunnel position to the anteromedial and posterolateral bundle femoral origins of the anterior cruciate ligament. Am J Sports Med 2008;36:73-79.

24. Tompkins M, Milewski MD, Carson EW, et al. Femoral tunnel length in primary anterior cruciate ligament reconstruction using an accessory medial portal. Arthroscopy 2013;29:238-243.

25. Almekinders LC, Chiavetta JB, Clarke JP. Radiographic evaluation of anterior cruciate ligament graft failure with special reference to tibial tunnel placement. Arthroscopy 1998;14:206-211.

26. Dejour H, Bonnin M. Tibial translation after anterior cruciate ligament rupture: Two radiological tests compared. J Bone Joint Surg Br 1994;76:745-749.

27. Tegner Y, Lysholm J. Rating systems in the evaluation of knee ligament injuries. Clin Orthop Relat Res 1985;198: 43-49.

28. Irrgang JJ, Anderson AF, Boland AL, et al. Development and validation of the International Knee Documentation Committee subjective knee form. Am J Sports Med 2001;29:600-613.

29. Buda R, Ruffilli A, Di Caprio F, et al. Allograft salvage procedure in multiple-revision anterior cruciate ligament reconstruction. Am J Sports Med 2013;41:402-410.

30. Webster KE, Feller JA, Kimp AJ, Whitehead TS. Revision anterior cruciate ligament reconstruction outcomes in younger patients: Medial meniscal pathology and high rates of return to sport are associated with third ACL injuries. Am J Sports Med 2018;46:1137-1142.

31. Kruse LM, Gray B, Wright RW. Rehabilitation after anterior cruciate ligament reconstruction: A systematic review. J Bone Joint Surg Am 2012;94:1737-1748. 
32. Janssen RP, du Mée AW, van Valkenburg J, et al. Anterior cruciate ligament reconstruction with 4-strand hamstring autograft and accelerated rehabilitation: A 10-year prospective study on clinical results, knee osteoarthritis and its predictors. Knee Surg Sports Traumatol Arthrosc 2013;21: 1977-1988.

33. Briggs KK, Lysholm J, Tegner $Y$, et al. The reliability, validity, and responsiveness of the Lysholm score and Tegner activity scale for anterior cruciate ligament injuries of the knee: 25 years later. Am J Sports Med 2009;37: 890-897.

34. Liechti DJ, Chahla J, Dean CS, et al. Outcomes and risk factors of rerevision anterior cruciate ligament reconstruction: A systematic review. Arthroscopy 2016;32: 2151-2159.

35. Muller B, Yabroudi MA, Lynch A, et al. Defining thresholds for the patient acceptable symptom state for the IKDC Subjective Knee Form and KOOS for patients who underwent ACL reconstruction. Am J Sports Med 2016;44: 2820-2826.

36. Wordeman SC, Quatman CE, Kaeding CC, Hewett TE. In vivo evidence for tibial plateau slope as a risk factor for anterior cruciate ligament injury: A systematic review and meta-analysis. Am J Sports Med 2012;40: 1673-1681.

37. Christensen JJ, Krych AJ, Engasser WM, et al. Lateral tibial posterior slope is increased in patients with early graft failure after anterior cruciate ligament reconstruction. Am J Sports Med 2015;43:2510-2514.
38. Lee CC, Youm YS, Do Cho S, et al. Does posterior tibial slope affect graft rupture following anterior cruciate ligament reconstruction? Arthroscopy 2018;34:2152-2155.

39. Dejour D, Saffarini M, Demey G, Baverel L. Tibial slope correction combined with second revision ACL produces good knee stability and prevents graft rupture. Knee Surg Sports Traumatol Arthrosc 2015;23:2846-2852.

40. Sonnery-Cottet B, Mogos S, Thaunat M, et al. Proximal tibial anterior closing wedge osteotomy in repeat revision of anterior cruciate ligament reconstruction. Am J Sports Med 2014;42:1873-1880.

41. Schillhammer C. Managing excessive posterior slope in anterior cruciate ligament reconstruction: Where do we draw the line? Arthroscopy 2018;34:2156-2157.

42. Hwang MD, Piefer JW, Lubowitz JH. Anterior cruciate ligament tibial footprint anatomy: Systematic review of the 21 st century literature. Arthroscopy 2012;28:728-734.

43. Group M. Meniscal and articular cartilage predictors of clinical outcome after revision anterior cruciate ligament reconstruction. Am J Sports Med 2016;44:1671-1679.

44. Anand BS, Feller JA, Richmond AK, Webster KE. Returnto-sport outcomes after revision anterior cruciate ligament reconstruction surgery. Am J Sports Med 2016;44:580-584.

45. Sonnery-Cottet B, Saithna A, Cavalier M, et al. Anterolateral ligament reconstruction is associated with significantly reduced ACL graft rupture rates at a minimum follow-up of 2 years: A prospective comparative study of 502 patients from the SANTI study group. Am J Sports Med 2017;45:1547-1557. 